\title{
Research on Dynamic Characteristics of Hard Cutting System
}

\author{
Yue Caixu, Fenghuize, Cai Chunbin, Zhang Haitao and Huang Cui \\ yuecaixu@163.com,fenghuize@gmail.com, \\ caichb@outlook.com,963057325@qq.com,851376422@qq.com \\ Harbin University of Science and Technology
}

\begin{abstract}
Tool wear is an inevitable and continuously aggravated process in precision hard cutting. Interaction between tool wear and dynamic characteristics in cutting system makes the machining accuracy and quality of machined surface declining, which effects dynamic characteristics of cutting system deteriorated significantly. Based on this, cutter dynamic modal parameters of a specific cutting system are measured in this paper. Cutting force and change law of acceleration power spectrum are studied by experiments. Influence mechanism of tool wear on system frequency energy distribution was obtained. The cutting system dynamic characteristics are quantified by establishing simulation model. Root locus plot of non-uniform load distribution is obtained and the minimum stiffness ratio in the root locus plot is determined. The effect of tool flank wear on cutting system stability in quantitatively stable is researched.The results can provide a theoretical basis for the selection of reasonable selection of stable precision cutting conditions in different tool wear conditions.
\end{abstract}

Keywords:Hard cutting process, Tool wear, Dynamic characteristics, Spectral density, Stable region

\section{Introduction}

Typical high strength steel is normally used in automobile manufacturing, mold and aerospace industry, such as steel and die steel, bearing steel and gear. The hardness of high strength steel can reach as high as HRC50-65 after quenching process [1,2]. In generally, rough machining of hardened steel workpiece is before quenching, and finishing grinding after quenching. The grinding has low efficiency, high cost, sever cutting liquid pollution, and high consumption of wheel and grinding fluid[3,4]. With the development of PCBN tool technology and application high performance machine tool system, it is a reality that hard cutting has been the final finishing method of hardened steel machining $[5,6]$. Compared with the traditional process of grinding, hardened steel hard cutting has high flexibility and processing efficiency, and can replace the grinding process as finish machining process in some machining occasions $[7,8]$.

Many domestic and foreign scholars have made abundant research on cutting system stability [9-10]. Typical achievements are as follows: Scholar M.S.Fofana got the expression of analyzing the flutter stability in cutting process by using typical Hopf bifurcation theory and CentreManifold theory and integral and Laplace transform[12]. British scholar F Abrai and others extended the equivalent orthogonal cutting conditions which applied to the cutting tool mechanical mode to dynamic cutting force, and presented a analysis model of dynamic cutting force and stability in ball-end milling[11]. $\mathrm{Wu}$ Weiguo from Jiangsu University et al, according to characteristics of precision machining center cutting slender parts, using the system dynamics and the linear stability theory, established the stability of the cutting system mathematical model, and based on this model he put forward the cutting parameters corresponding to stability threshold[12].Professor Zhao Feng took CA6136 lathe as the object of study, analyzed cutting stability through cutting vibration test, studied the mechanism of the regenerative chatter which was most often appear in cutting, and they obtained cutting stability of the lathe lobes diagram according to the experimental data [13].Dr Chen Guangjun from Harbin University from Science and Technology Systematically studied PCBN tool 
stability in hardened steel cutting, and obtained steady state cutting conditions in specific cutting environment. Because the tool wear is inevitable and continuous increase, the occurrence of tool wear will have a significant impact on the stability of the system. so the research on tool wear and dynamic cutting system has more necessity.

According to the cutting system stability, domestic and foreign scholars have made many achievements, but few scholars studied the dynamic characteristics of cutting system on the basis of tool wear factors. Based on this, this paper firstly tests the frequency response characteristics of cutter dynamic cutting system, and reveals the effect of tool wear mechanism on the vibration characteristics of the system. The research results can provide the theoretical basis for reasonable selection results for precision stable cutting conditions in different tool wear conditions.

\section{Hard Cutting Experimental Equipment and Related Materials}

In the stable cutting conditions, the vibration cutting system is focus on forced vibration, vibration signal has a frequency band high frequency range, the typical characteristics of frequency domain energy dispersion. When the cutting chatter occurs in cutting system, the main position of self-excited vibration strong regeneration will occupy the vibration signal energy, frequency domain energy concentrated in the vicinity of cutting vibration system inherent low frequency. In order to analyze the effect of tool wear on the machining system stability, this study sets up the test platform of cutting system stability, as shown in Fig.1. The test site cutting tool modal parameters as shown in Fig.2,the test instrument production model for Donghua company for the DH5922 data acquisition system and DHMA modal analysis software.The cutting tool is PCBN tool, and the material of workpiece is $\mathrm{Cr} 12 \mathrm{MoV}$.

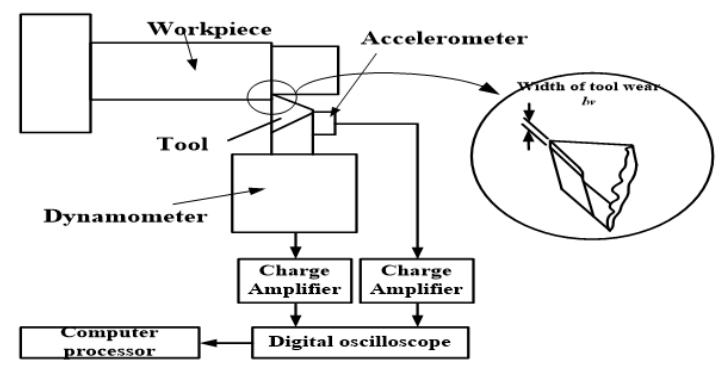

Figure 1. Test Platform Built on Stability of Cutting Systems

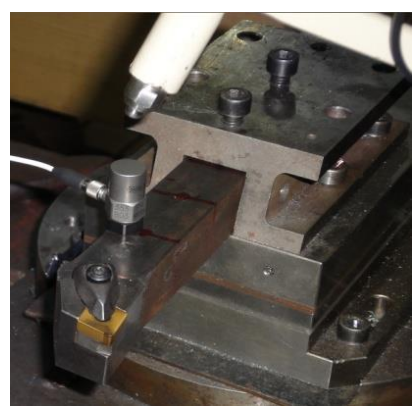

Figure 2. Modes of Cutting Tool System

To test node division of the tool/toolholder system, this experiment adopts hammer excitation and PCB acceleration sensor. Modal test for tool handle according to frequency response function method was done, then the test system schematic diagram was obtained. Simulation and experiment results of modal parameters for tool system are shown in Fig.3 and Fig.4. 


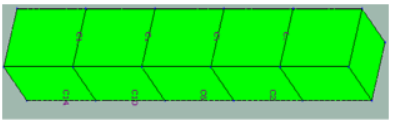

(a)

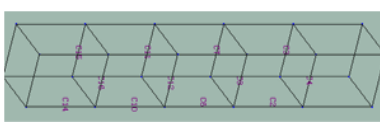

(b)

Figure 3. Cutting Tool Grid

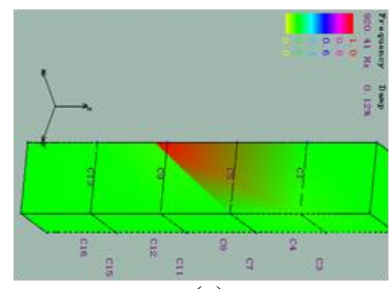

(a)

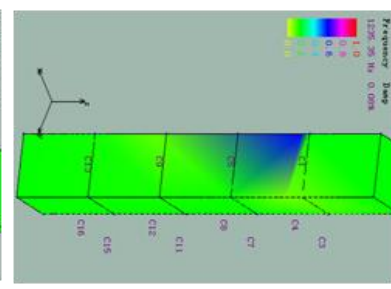

(b)

\section{Figure 4. Modal Parameter Simulation Results of Tool System}

Vibration of tool has certain frequency in vibration exciter excitation, and the signals of tool rod vibration response was picked up by the acceleration sensor. Establishment of test system is preparation process of modal test. It mainly includes: theory analysis and calculation of measured object, determining test plan, including determination of the excitation mode, sensor selection, data collection and analysis instrument selection. In accordance with program requirements of installation and commissioning test, the measured frequency response is shown in Fig.5.

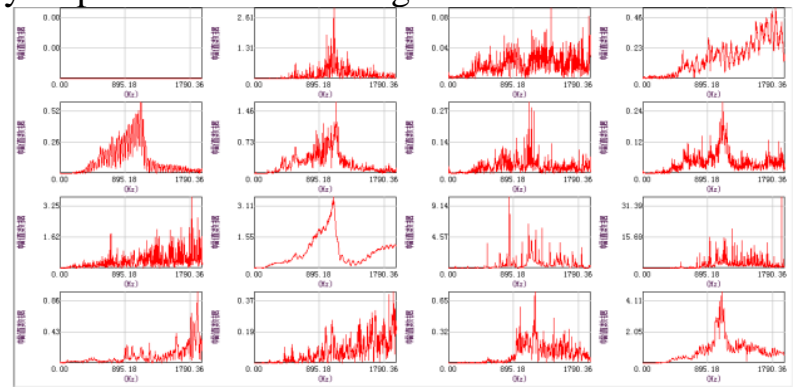

Figure 5. Measured Frequency Response Diagram of Cutting Tool System

In the premise of selecting different modes normalized criteria, the modal mass and modal stiffness can be calculated. The test results show that the first order natural frequency of system is $532.23 \mathrm{~Hz}$. The damping ratio is $0.25 \%$, and the second order natural frequencies of the system is $920.41 \mathrm{~Hz}$. The damping ratio is $0.08 \%$.In the same normalized criteria, modal mass and modal parameters calculation method of rigidity is consistent.

\section{Effect of Tool Wear on the Cutting System Dynamic Characteristics}

In this study, cutting experiments were done when tool wear is $0 \mathrm{~mm}, 0.2 \mathrm{~mm}$ and $0.3 \mathrm{~mm}$ respectively. The cutting conditions is following as cutting speed is $234 \mathrm{~m} / \mathrm{min}$, cutting feed rate is $0.1 \mathrm{~mm} / \mathrm{r}$, and cutting depth is $0.2 \mathrm{~mm}$. The experiment separately collected radial cutting force and acceleration signal acquisitio, as shown in Fig.6 and Fig.7. From the results, in the same cutting conditions, with tool wear increases, the cutting force increased significantly, also the cutting acceleration signal volatility. The reason is that increase of tool flank friction effect, quality of machined surface become worse. The interact force between tool and workpiece become vibration source, which make force vibration more strongly. 

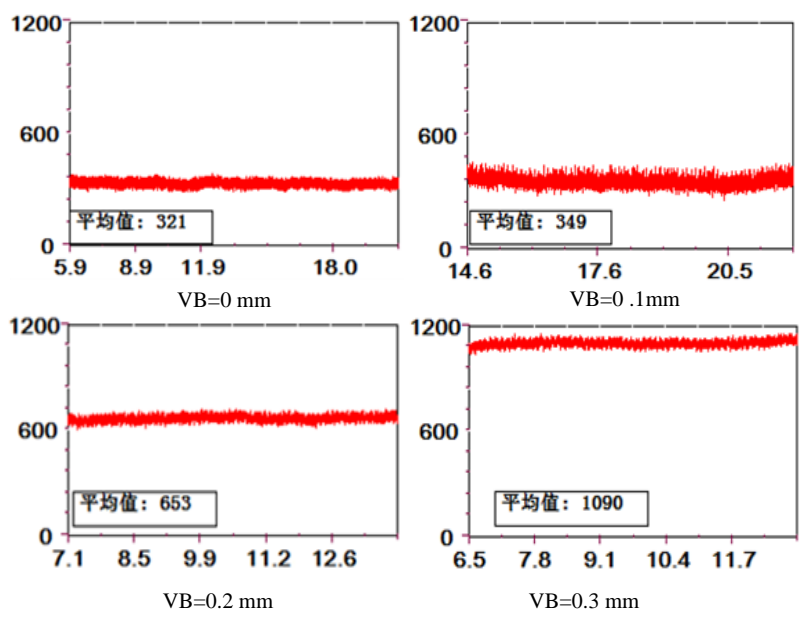

Figure 6. Cutting Force Signal under Different Conditions
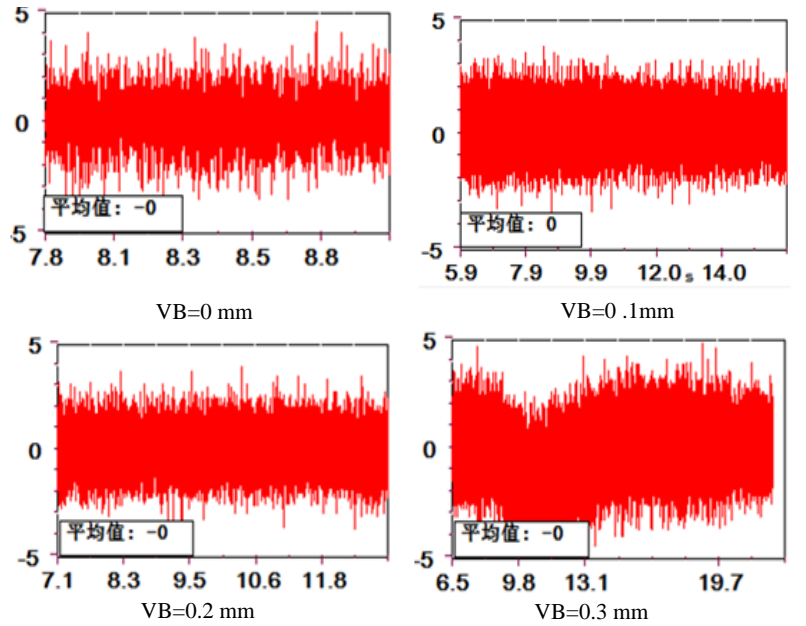

Figure 7. Acceleration Signal under Different Conditions
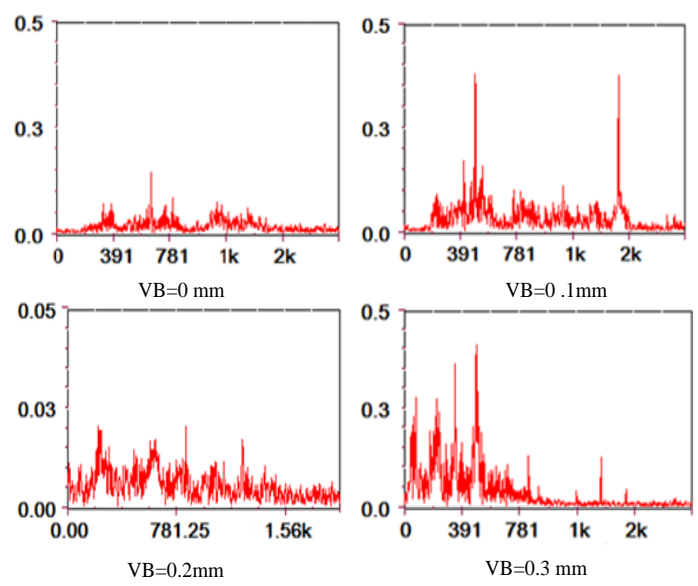

Figure 8. Cutting Force Power Spectrum 


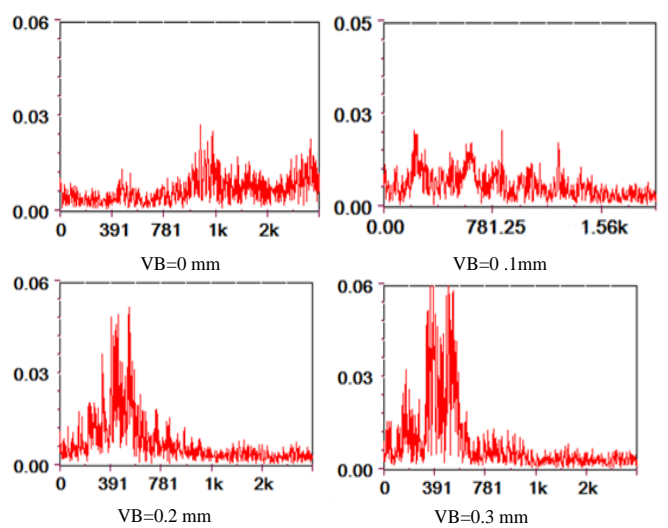

\section{Figure 9. Acceleration Power Spectrum Signal when Different Tool Wear}

Fig.8 and Fig.9 are respectively cutting force and acceleration power spectrum of signal power spectrum under different tool wear conditions. Test results show that the small amplitude vibration still exists under stable cutting conditions. When the tool wear not or wear volume is small, the system oscillation frequency is mainly distributed near $532 \mathrm{~Hz}$, and natural frequency of the tool system occupies the main status. With increase of tool wear low frequency vibration increased significantly.

\section{Research on Dynamic Characteristics base on Spectral Sensity}

In order to establish a single degree of freedom linear model containing tool wear, in this study, we assuming that the workpiece is a rigid body and tool is flexible body. The tool goes along a unique direction $\mathrm{X}$ to vibration. The feed direction is along the radial direction of workpiece and parallel to the $\mathrm{X}$ direction. Even if the tool deviate from the tangential and axial direction, turning operations are only effected by the radial vibration of the tool. Therefore, a single degree of freedom vibration model can be assumed instead of 3 degrees of freedom model. As the cutting point of radius and not cutting thickness values compared to is very small, so we ignore the effects of geometrical size of tool in in hard cutting. Tool edge position is set to the zero point of the $\mathrm{X}$-axis. Cutting force $\mathrm{F}$ in the $\mathrm{X}$ direction component $\mathrm{Fx}$ is balance with the spring force. The movement formula in the $\mathrm{X}$ direction as :

$m \ddot{x}(\mathrm{t})+\mathrm{c} \dot{\mathrm{x}}(\mathrm{t})+\mathrm{k}_{\mathrm{m}} x(\mathrm{t})=\Delta \mathrm{F}_{\mathrm{x}}(\mathrm{t})$

Here $\mathrm{m}$ is the mass of the tool, $\mathrm{c}$ is the coefficient of viscosity, $\mathrm{km}$ is the structure stiffness, and $\triangle \mathrm{Fx}(\mathrm{t})$ is the amount of change of the cutting forces in $\mathrm{X}$ direction.

In Eq. $1, \Delta \mathrm{Fx}(\mathrm{t})$ is composed of a continuous rotation of variables in cutting process which is caused by without resection of part thickness changes, As shown in Fig. 10. When tool wear is increasing, non-uniform load distribution $6(\mathrm{ZVB})$ along the ZVB coordinate distribution.

$$
\sigma\left(\mathrm{z}_{l}\right)=\frac{1}{V B}\left[1+\cos \left(\pi\left(\frac{\mathrm{z}_{V B}}{V B}\right)^{\mathrm{n}_{\mathrm{s}}}\right)\right], z_{V B} \in[-V B, 0]
$$

Here VB is the width of the amount of wear, ns is decided by the amount of plastic deformation and assuming that is inversely proportional to amount of wear:

$n_{s}=\alpha / V B$

Where $\alpha$ is normal number. Load distribution in a maximum wear beginning has a maximum, place at the end of the wear becomes zero, As shown in Fig.11. Load distribution characteristics of tool wear on the workpiece can reflect the influence of plastic deformation degree. Therefore, Impact and wear of plastic deformation on the flutter stability can reaction through the load distribution. Considering the initial 
conditions and re-computing the upper and lower limits, we can obtain the characteristic equation about the amount of wear of the tool with a non-uniform load distribution as:

$$
s^{2}+2 \zeta \omega_{n} s+\omega_{n}^{2}+k_{c} / k_{m} \omega_{n}^{2}\left(1-e^{-s T_{p}}\right)=0
$$

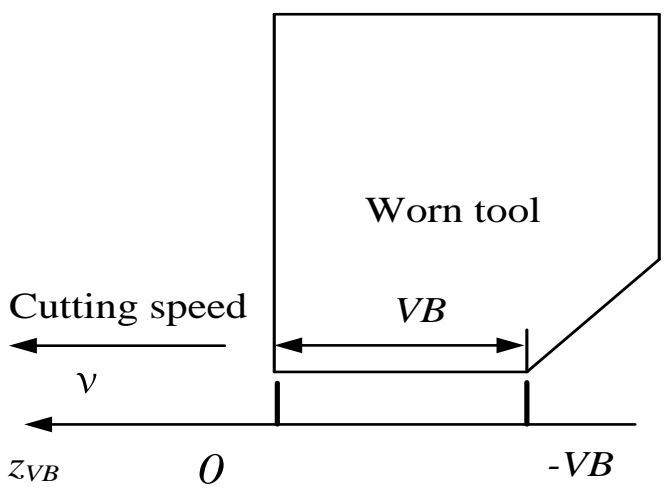

Figure 10. Diagram of Coordinates ZVB and Wear VB

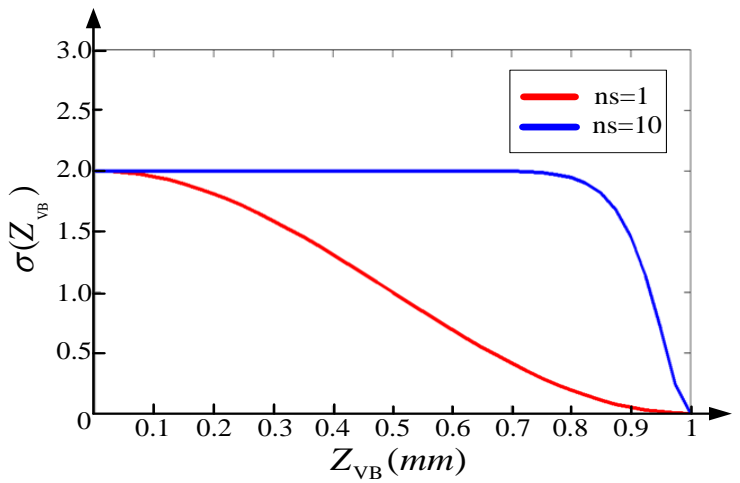

Figure 11. Non-uniform Load Distribution

\section{Research on Stability of Cutting System Limit Considering Tool Wear}

Foreign researchers Baradie EI putted forward the method to simulate the application of computer simulation to predict stability limit of machine tool cutting system, and accordingly selected stable cutting parameters and made the cutting process in the stable region, the research in this field has been made certain achievements. This paper will focus on the critical parameters obtained by studying the closed-loop flutter stability in the roots of the characteristic equation of the situation. If all the roots of the closed-loop characteristic equation have negative real roots, the system is in steady state. The critical parameters of the flutter stability can draw an item of the stiffness ratio of the root locus to accurately determine. The closed-loop characteristic equation of non-uniform load distribution can be derived expressions for the stiffness ratio:

$$
\frac{k_{c}}{k_{m}}=\frac{s^{2}+2 \zeta \omega_{n} s+\omega_{n}^{2}}{\omega_{n}^{2} \int_{-h}^{0} \sigma(\theta) e^{s \theta_{d}} d \theta\left(e^{-s T_{p}}-1\right)}
$$

While $w n=111 \mathrm{~Hz}, \xi=0.054$, based on the root locus of the closed-loop system, we can find the different values of $n$. As shown in Fig.12. From Fig.13, while $n=1, n=2$, we can see that when the branch decision value $\mathrm{n}$ is greater than or equal to 2 . The branch will be 
entered into the imaginary axis. When a branch into the imaginary axis, the other branch in the left side of the s-plane, the stiffness ratio of the value corresponds to the stability of the system on the imaginary axis. Then, the minimum stiffness ratio is a critical parameter that determines the stability of the system.

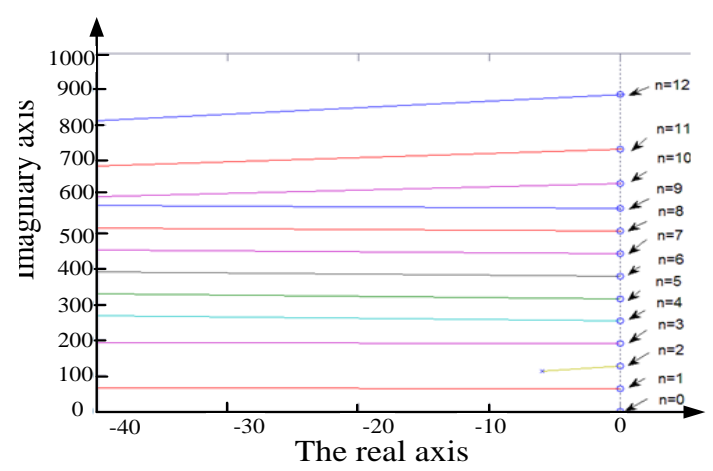

Figure 12. Root Locus Diagram of Non Uniform Distribution

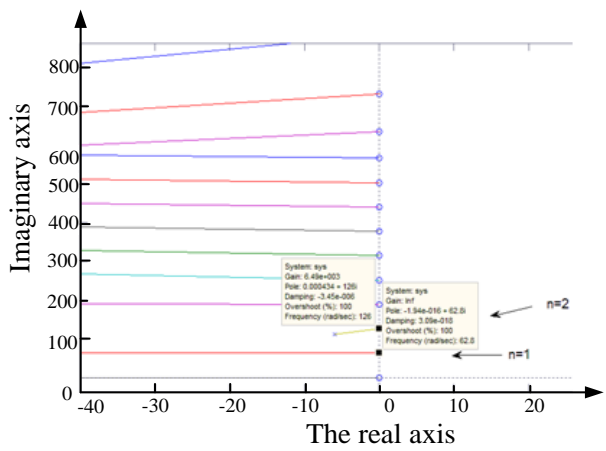

Figure 13. Data Display of Root Locus Diagram Load

In this paper, with the value of stiffness ratio increases, branch will enter the s plane of the right, into the positive region. Therefore, while $w n=111 \mathrm{~Hz}, \xi=0.054$, we can find the minimum stiffness ratio on the root locus of the closed-loop system, as shown in Fig.14. Through the calculation of the imaginary axis stiffness than the value, we can obtain from the beginning of 2 growth $n$. Under this condition, on the basis of numerical calculation, $\mathrm{n}=2$ branch in the imaginary axis stiffness ratio of the minimum value. And the minimum value of corresponding frequency value for flutter frequency and larger than the natural circular frequency.

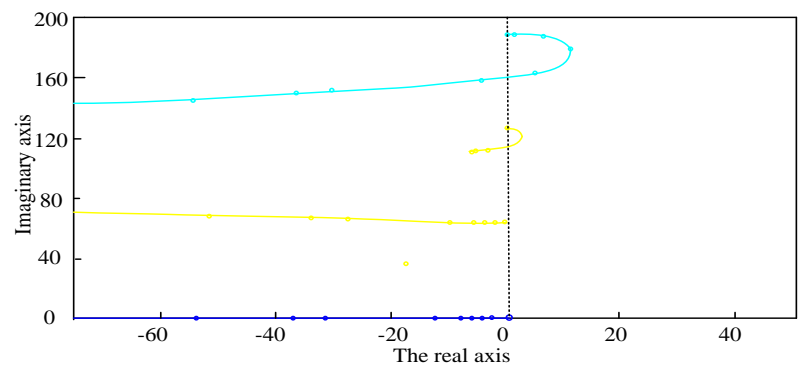

Figure 14. Determination of Minimum Stiffness Ratio in Root Locus Diagram

Analysis of special cases is regarded as the stability of tool wear analysis tool to derive stability. When ns is equal to 1 , and wear length VB in non-uniform load distribution $\sigma$ ( $\theta$ )is small enough to be ignored load distribution becomes $\delta(\theta)$. The results show the 
tool value and zero position for the tool wear are the same.

If $\omega^{2}-\omega_{n}\left(1+k_{c} / k_{m}\right)+2 \xi^{2} \omega_{n}^{2}>0$, the system with time delay T becomes unstable.

\section{Prediction of Stability Limits}

In hard cutting process, this study measured by different cutting speed system stiffness to consider the effect of tool wear on the system stability region. Because the cutting width is a close to the actual parameter in the cutting operation, so it can be used to replace the stability diagram of cutting width ratio of stiffness. The following conversion formula:

$$
w o c=(0.031 \Omega+2.6)\left(k_{c} / k_{m}\right)+(0.02 \Omega-0.3)
$$

Here, woc is the cutting width. Because the stiffness ratio of the minimum value in the stability analysis is a critical stability parameter, the minimum stiffness ratio above the region is known as the unstable region. The minimum value below the region is known as the stability region and the stable region is not obvious for the cutting speed flutter. For the non-uniform load distribution, this study selected the research value of $0 \mathrm{~mm}, 0.1 \mathrm{~mm}$ and $0.2 \mathrm{~mm}$ of the width of different tool wear, tool wear of cutting system stability limit change as shown in Fig.15. By comparing the different tool wear stability limit change amount of wear, that has a significant impact on the stability. When the flank wear is not more than $0.2 \mathrm{~mm}$, increase of tool wear volume will lead to stable regional stability limit line broadening. With increase of wear length, under the stable line area increase. At the same time, effect the stability of cutting system mainly occurs in the high-speed range.

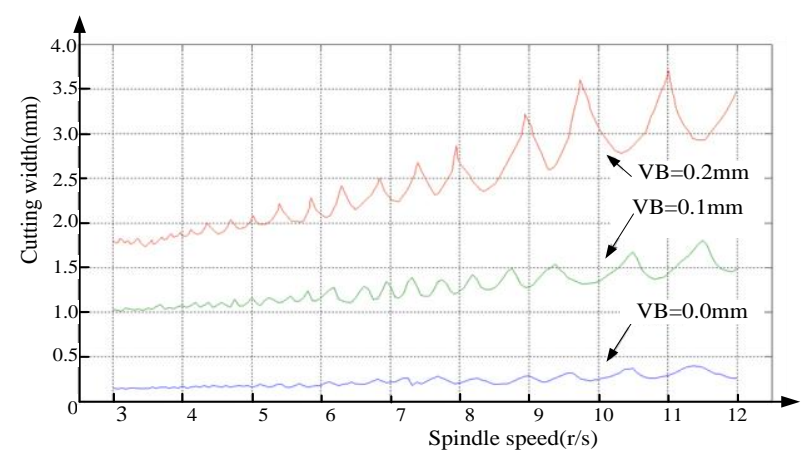

Figure 15. Stability Region Limiting under Different Tool Wear in Diagram

\section{Conclusion}

Cutting force and vibration characteristics research results show that small amplitude vibration still exists under stable cutting conditions. When tool wear not or wear volume is small, the system oscillation frequency is mainly distributed near $532 \mathrm{~Hz}$, and natural frequency of the tool system occupies the main status. With increase of tool wear low frequency vibration increased significantly.

With increase of tool wear, cutting force fluctuation in the time space gradually increased. Simulation model evaluate different tool wear stability state of the system, and the simulation results show that there is a close relationship between the stability of tool wear in cutting process and machining system.

With aggravation of tool wear, cutting system damping become larger, and stability of cutting system increase. When cutting speed and tool wear increases, stability of process system becomes better. If the tool flank wear is not greater than $0.2 \mathrm{~mm}$, increase of tool wear volume will lead to stable regional stability limit line broadening. At the same time, 
effect the stability of cutting system mainly occurs in the high-speed range.

\section{Acknowledgement}

The authors gratefully acknowledge the support of Natural Science Foundation of Heilongiiang Province(E201307) and National Natural Science Foundation of China((51105119).

\section{Reference}

[1] R. T. Coelho, E. G. Ng and M. A. Elbestawib, "Tool wear when turning hardened AISI 4340 with coated PCBN tools using finishing cutting conditions", International Journal of Machine Tools and Manufacture, vol. 47(, no. 2, (2007), pp. 263-272.

[2] X. L. Liu, H. M. Pen and T. Chen, "Effect of different edge preparation on high speed turning hardened steel process", Proceedings of the 12th International Conference on IMCC, Xi'an, China, Materials Science Forum, (2006).

[3] F. Hashimotoa, Y. B. Guo and A. W. Warren, "Surface Integrity Difference between Hard Turned and Ground Surfaces and Its Impact on Fatigue Life", CIRP Annals - Manufacturing Technology Volume, vol. 55 , no. $1,(2006)$, pp. $81-84$.

[4] M. R. Outeiro and J. C. Chandrasekaran, "A review of surface integrity in machining and its impact on functional performance and life of machined products", Sustainable manufacturing, International Journal of Sustainable Manufacturing, vol. 1, no. 1-2, (2008), pp. 203-236.

[5] Z. Q. Liu and Q. Liu Q, "Solution and analysis of chatter stability for end milling in the time-domain", Chinese Journal of Aeronautics, vol. 2, no. 21, (2008), pp. 169-178.

[6] L. Filice, D. Umbrello, F. Micari and L. Settineri, "On the Finite Element Simulation of Thermal Phenomena in Machining Processes", Advanced Methods in Materia, (2007), pp. 263-278.

[7] E. Soils, C. R. Peres and J. E. Jimenez, "A new analytical-experimental method for the identification of stability lobes in high-speed milling”, International Journal of Machine Tools and Manufacture, vol. 44, no. 15, (2004), pp. 1591-1597.

[8] Z. Q. Liu and Q. Liu, "Solution and analysis of chatter stability for end milling in the time-domain", Chinese Journal of Aeronautics, vol. 2, no. 21, (2008), pp. 169-178.

[9] W. X. Tang, X. Ai and S. Zhang, "Dynamic modeling for high-speed milling system with centrifugal force and gyroscopic effect", Key Engineering Materials, (2004), pp. 258-259,848-852.

[10] M. S. Fofana, "Effect of regenerative process on the sample stability of a multiple delay differential equation", Chaos, Solitons and Fractals, vol. 14, (2002), pp. 301-309.

[11] F. Abrai, M. A. Elbestaw and I. A. D. Spence, "On the Dynamics of Ball End Milling Modeling of Cutting Forces and Stability Analysis", Int .J. Mach. Tools Manufact, vol. 38, no. 3, (1998), pp. 215-237.

[12] W. G. Wu, G. C. Wang, C. G. Shen and L. J. Ma, "Effect of spindle dynamics on system stability insuper-precision machining center", vol. 28, no. 4, (2007), pp. 293-296.

[13] Z. Feng, "Study on the stability of system of the lathe cutting", CAD/CAM and manufacturing information, vol. 7, (2012), pp. 77-80. 
International Journal of $\mathrm{u}-$ and $\mathrm{e}-$ Service, Science and Technology Vol.8, No.3 (2015) 